\title{
Quantum caging in interacting many-body all-bands-flat lattices
}

\author{
Carlo Danieli $\odot,{ }^{1,2}$ Alexei Andreanov $\odot,{ }^{2,3}$ Thudiyangal Mithun $\odot,{ }^{2,4}$ and Sergej Flach ${ }^{2,3}$ \\ ${ }^{1}$ Max Planck Institute for the Physics of Complex Systems, Dresden D-01187, Germany \\ ${ }^{2}$ Center for Theoretical Physics of Complex Systems, Institute for Basic Science (IBS), Daejeon 34126, Korea \\ ${ }^{3}$ Basic Science Program (IBS School), Korea University of Science and Technology (UST), Daejeon 34113, Korea \\ ${ }^{4}$ Department of Mathematics and Statistics, University of Massachusetts, Amherst, Massachusetts 01003-4515, USA
}

(Received 30 April 2020; revised 4 January 2021; accepted 20 July 2021; published 17 August 2021)

\begin{abstract}
We consider translationally invariant tight-binding all-bands-flat networks which lack dispersion. In a recent work [C. Danieli et al., Phys. Rev. B 104, 085131 (2021)], we identified the subset of these networks that shows nonlinear caging in the presence of Kerr-like local nonlinearities, i.e., it preserves nonexcited network sites and therefore keeps compact excitations compact. Here we replace nonlinear terms by Bose-Hubbard interactions and study quantum caging. We identify the quantum caging conditions that are related to the nonlinear caging conditions and that guarantee the existence of an extensive set of conserved quantities in any lattice dimension, as first revealed in Tovmasyan et al. [Phys. Rev. B 98, 134513 (2018)] for a set of specific networks. Consequently, transport is realized through moving pairs of interacting particles that break the single-particle caging. We further prove the existence of degenerate energy renormalized compact states for any finite number $M$ of participating particles in one- and higher-dimensional lattice-results that explain and generalize previous observations for two particles on a diamond chain [Vidal et al., Phys. Rev. Lett. 85, 3906 (2000)].
\end{abstract}

DOI: 10.1103/PhysRevB.104.085132

\section{INTRODUCTION}

The study of localization phenomena in systems of interacting particles gave rise to some of the most remarkable research streams in condensed-matter physics during the past decades. Typically, these phenomena arise in the absence of translation invariance, as both the first prediction of singleparticle localization [1,2] and the finite-temperature transition to many-body localized phases of weakly interacting quantum particles $[3,4]$ have been obtained in tight-binding networks in the presence of uncorrelated spatial disorder. However, both single-particle and many-body particle localization can be achieved in translationally invariant setups (see, e.g., Refs. [5,6] for a discussion on disorder-free many-body localization).

One of the notable examples of single-particle localization in translationally invariant lattices are flatband networkslattices where at least one of the Bloch energy bands is independent of the wave vector (hence, dispersionless or flat) [7-9]. Differently from Anderson localization, where the disorder causes all the eigenstates to become exponentially localized, the eigenstates associated with a flatband have strictly compact support spanned over a finite number of unit cellsand therefore they are called compact localized states (CLS). Since their first appearance as mathematical testbeds for ferro-

Published by the American Physical Society under the terms of the Creative Commons Attribution 4.0 International license. Further distribution of this work must maintain attribution to the author(s) and the published article's title, journal citation, and DOI. Open access publication funded by the Max Planck Society. magnetic ground states $[10,11]$, the extension of the flatband concept to novel lattice geometries has been of crucial interest. This gave rise to several generator schemes [12-17] adopting different principles to design artificial flatband lattices in different spatial dimensions.

If all Bloch bands are flat, then all single-particle eigenstates are spatially compact. In this all bands flat (ABF) case, single-particle transport is fully suppressed and noninteracting particles remain caged within a finite volume of the system. This caging phenomenon due to the collapse of the Bloch spectrum was originally dubbed Aharonov-Bohm (AB) caging since it emerged from a fine-tuning of a magnetic field into a time-reversal symmetry invariant case. AB caging was first introduced in a two-dimensional Dice lattice structure [18]. From the first case, the study of this localization has been further extended [19-24] while experimentally AB caged systems have been realized in photonic lattices $[25,26]$ and qubits nanocircuits [27].

Since an ABF model has all bands flat, the system displays time-reversal invariance. Therefore, there is no need to search for ABF models by detouring via time-reversal broken systems with nonzero magnetic synthetic fluxes or simply magnetic fields, only to rearrive back to a time-reversal symmetry invariant case. Instead, it is much simpler to fine-tune time-reversal symmetry invariant tight-binding manifolds in order to isolate the ABF cases. This task was executed in Ref. [28] through a set of local unitary detangling transformations and their inverse entangling ones, leading to a generator of $\mathrm{ABF}$ models in any lattice dimension with any number of flatbands. The detangled basis is the preferred one for the study of perturbations such as disorder and interactions.

The impact of interactions on caged particles was studied in the one-dimensional (1D) Creutz lattice [29-34] and the 
2D Dice lattice [35]. In both of these cases as well as in the 1D AB diamond (rhombic) lattice, it has been shown that the Hubbard interaction breaks the single-particle caging for some eigenstates by inducing transporting bound states of pairs of particles $[34,36]$. Transporting bound states coexist with the trivial two-particle states with particles caged in two CLS which are separated beyond the reach of the Hubbard interaction and which therefore remain exact eigenstates of the two-particle system. Remarkably, some macroscopically degenerate eigenstates continue to lack dispersion but show energy renormalization upon tuning the Hubbard interaction strength [36].

In a related work in Ref. [28] we introduced an ABF generator scheme in any lattice dimension. Each set of ABF models is related to one detangled $\mathrm{ABF}$ lattice. For a given member of the set, additional short-range nonlinear interactions destroy caging in general and induce transport. However, finetuned subsets of ABF lattices enable caging to be completely restored. We derived necessary and sufficient fine-tuning conditions for nonlinear caging including computational evidences.

In this work, we replace nonlinear terms by Bose-Hubbard interactions and study quantum caging phenomena. We prove that in the quantum case, the fine-tuning conditions for nonlinear caging imply the existence of an extensive set of conserved local number parity operators-quantities first revealed in Ref. [34] for a set of specific one-dimensional networks. Furthermore, we show that the conserved quantities lead to the existence of macroscopically degenerate interaction renormalized compact states for any finite number $M$ of participating particles. Our results apply to lattices of any spatial dimension, explaining and generalizing previous observations for two particles in the diamond chain [36].

\section{SINGLE-PARTICLE DETANGLING AND ENTANGLING: ABF GENERATOR SCHEME REVISITED}

In a related work in Ref. [28] it was shown that a singleparticle ABF network can be detangled and entangled with local unitary transformations, arriving at a generator of $\mathrm{ABF}$ networks. Assuming a translationally invariant tight-binding network, a local unitary transformation set $U_{1}$ is given by a product of commuting local unitary transformations (LUTs). Each LUT acts on a spatially localized Hilbert subspace, and all LUTs can be obtained from one by all possible discrete space translations. Given a $1 \mathrm{D} \mathrm{ABF}$ network with short-range hopping, it was shown [28] that a finite number of noncommuting LUT sets $U_{1}, \ldots, U_{u}$ will detangle the Hamiltonian into a completely diagonal form. The number $(u+1)$ corresponds to the hopping range. Inverting this procedure leads to the most general and exhausting $\mathrm{ABF}$ generator with the elements of the LUTs being the relevant control parameters. Extensions to higher lattice dimensions $d=2,3, \ldots$ result in ABF generators with the number of unitary sets increasing $\sim d(u+1)$.

With $v$ flatbands $\left\{E_{a}\right\}_{a \leqslant v}$, the detangled parent Hamiltonian is diagonal with

$$
\hat{\mathcal{H}}_{\mathrm{fd}}=\sum_{k} \hat{f}_{k}
$$

with each local Hamiltonian $\hat{f}_{k}$ being diagonal and of rank $v$. All local Hamiltonians have identical eigenvalue sets $\left\{E_{a}\right\}_{a \leqslant n u}$ corresponding to the spectrum of the Hamiltonian (1). The corresponding eigenvectors are compact localized states in real space. The first entangling step consists of applying one LUT $U_{1}$. The resulting family of ABF Hamiltonians is coined semidetangled (SD) and is a sum over commuting local Hamiltonians [37]

$$
\hat{\mathcal{H}}_{\mathrm{sd}}=\sum_{k} \hat{s}_{k}
$$

with each $\hat{s}_{k}$ of rank $v$. The system still shows trivial compact localization-dynamics is restricted to each of the $v$-mers related to a specific $\hat{s}_{k}$. Adding $d$ (noncommuting) LUTs $U_{2} \cdots U_{d+1}$ results in a nontrivial entangling and full connectivity on the entire network. The choice of the LUTs and their matrix elements fix one particular ABF family member.

\section{MANY-BODY INTERACTIONS: NECESSARY AND SUFFICIENT CONDITIONS FOR THE EXISTENCE OF LOCAL CONSERVED QUANTITIES}

We choose a particular ABF family member and add onsite Bose-Hubbard interaction

$$
\hat{\mathcal{H}}_{1}=\frac{U}{2} \sum_{n \in \mathbb{Z}, a} \hat{c}_{n a}^{\dagger} \hat{c}_{n a}^{\dagger} \hat{c}_{n a} \hat{c}_{n a},
$$

where $\hat{c}_{n, a}$ denote bosonic annihilation operators in a unit cell $n$ with $1 \leqslant a \leqslant v$. The replacement of the operators by $c$-numbers was considered in Ref. [28], which addressed the question of whether there is an ABF family submanifold that preserves nonlinear caging. The submanifolds were derived by transforming an ABF family member back into its detangled parent basis.

For the quantum counterpart, such a transformation turns the Bose-Hubbard interaction into

$$
\hat{\mathcal{H}}_{1}=\frac{U}{2} \sum_{n a, m b ; k c, l d} V_{n a, m b ; k c, l d} \hat{d}_{n, a}^{\dagger} \hat{d}_{m, b}^{\dagger} \hat{d}_{k, c} \hat{d}_{l, d}
$$

in the detangled representation. Here the operators $\hat{d}_{n, a}$ are the annihilation operators in the detangled basis. These terms represent generic hopping of pairs of particles, implying that without any fine-tuning, either of the interaction or the singleparticle Hamiltonian, the caging is broken and there should be transport in the interacting problem.

This is in accordance with the previous studies that indicate that we should expect the emergence of transporting states: Vidal et al. [36] predicted extended states in the AharonovBohm diamond chain with the Hubbard interaction already for two particles. Tovmasyan et al. [34] confirmed that and furthermore conjectured the existence of an extensive set of conserved quantities - number parity operators - to be a generic feature of interacting ABF networks based on several models considered.

We now provide a proof of this conjecture along with the necessary and sufficient conditions on the interactions for the existence of an extensive set of conserved quantities. We also discuss potential generalizations of this result. The proof follows very naturally from our results for classical 
nonlinear interactions: conserved quantities-number parity operators-appear every time the interacting AFB network only allows particles to move in pairs between the unit cells, or, equivalently, whenever the classical version of the network features caging. Indeed, as we showed in Ref. [28], nonlinear caging occurs in classical models iff the interaction, in the detangled basis, takes the following form (for the Kerr-like nonlinearity, which corresponds to the Hubbard-like interaction in the quantum case):

$$
\begin{aligned}
\hat{\mathcal{H}}_{1}= & \frac{U}{2} \sum_{n a, b ; m c, d} V_{n a, n b ; m c, m d} \phi_{n, a}^{*} \phi_{n, b}^{*} \phi_{m, c} \phi_{m, d} \\
& +\frac{U}{2} \sum_{n a, b ; m c, d} V_{n a, m b ; n c, m d} \phi_{n, a}^{*} \phi_{m, b}^{*} \phi_{n, c} \phi_{m, d},
\end{aligned}
$$

where $\phi_{n, a}$ is a classical amplitude on the site $a$ inside unit cell $n$. The exact choice of the interaction is not relevant for the proof. The quantum version of this Hamiltonian in the second quantized form reads

$$
\begin{aligned}
\hat{\mathcal{H}}_{1}= & \frac{U}{2} \sum_{n a, b ; m c, d} V_{n a, n b ; m c, m d} \hat{d}_{n, a}^{\dagger} \hat{d}_{n, b}^{\dagger} \hat{d}_{m, c} \hat{d}_{m, d} \\
& +\frac{U}{2} \sum_{n a, b ; m c, d} V_{n a, m b ; n c, m d} \hat{d}_{n, a}^{\dagger} \hat{d}_{m, b}^{\dagger} \hat{d}_{n, c} \hat{d}_{m, d} \\
= & \hat{\mathcal{H}}_{\mathrm{p}}+\hat{\mathcal{H}}_{\mathrm{dd}} .
\end{aligned}
$$

Such interaction, which we loosely term a quantum caging one, only allows particles to hop in pairs between the unit cells. The terms in the above two sums have an immediate interpretation: the first sum $\hat{\mathcal{H}}_{\mathrm{p}}$ only contains terms that allow pairs of particles to hop from one unit cell to another, while the terms in the second sum $\hat{\mathcal{H}}_{\mathrm{dd}}$ move two particles, one within unit cell $n$ and the other within unit cell $m$. In other words, the terms in the first sum can only change the number of particles in a unit cell by 2, while the terms in the other sum cannot change this number. Since the single-particle Hamiltonian in the detangled basis does not move particles at all, the full Hamiltonian commutes with the parity operator of the number of particles in each unit cell [34] (the full proof is provided in Appendix A):

$$
\hat{C}_{n}=\exp \left[i \pi \sum_{a} \hat{d}_{n, a}^{\dagger} \hat{d}_{n, a}\right] .
$$

This proves the sufficiency of the condition, while the necessity is self-evident. The operator $\hat{C}_{n}$ has only two eigenvalues $c_{n}= \pm 1$ corresponding to the even $\left(c_{n}=1\right)$ or odd $\left(c_{n}=-1\right)$ occupation of the unit cell $n$ [34]. Consequently, there can be caged isolated particles in the system, since for an odd number of particles in a unit cell, one particle is doomed to stay in that unit cell forever, since the interaction is unable to move it [34]. Note also that a system of spinless fermions freezes completely for a quantum caging interaction, since double occupancy is forbidden.

Are there any additional caging features in ABF systems induced by this class of interaction terms? Such a question appears reasonable following the prediction of nondispersive states of two interacting spinful fermions in the AharonovBohm diamond chain [36]. Note that the cases considered in
Ref. [34] correspond to a fine-tuning of the 1D models where particles have to move in pairs between the unit cells (in the detangled representation). The observation of similar features for the 2D Dice lattice [34] provides indirect evidence in support of our conjecture that $\mathrm{ABF}$ lattices in any dimension can be detangled by local unitary transformations [28].

We note that it is also possible to perform further finetuning of the interaction and eliminate the interaction-assisted hopping of pairs, and turn the second sum into a pure densitydensity interaction, leading to no particle transport, e.g., a perfect charge insulator [34,37-39]. Finally, just like in the classical case, we can use this construction to test whether a given combination of an ABF network and many-body interaction possesses conserved quantities - by inspecting the interaction in the detangled basis. We can also invert the procedure and start with a given interaction that moves particles in pairs in the detangled basis, and then apply a unitary transformation and get into some other basis, resulting in general in a model with complicated many-body interactions that possesses an extensive set of conserved quantities.

\section{RENORMALIZED COMPACT STATES OF INTERACTING PARTICLES}

Let us focus on the class of interacting $\mathrm{ABF}$ networks admitting an extensive set of conserved quantities $\hat{C}_{n}$ in Eq. (7) and consider their Hamiltonian $\hat{\mathcal{H}}=\hat{\mathcal{H}}_{\mathrm{fd}}+\hat{\mathcal{H}}_{1}$ expressed by the single-particle part $\hat{\mathcal{H}}_{\mathrm{fd}}$ in the detangled representation (1) and the interaction $\hat{\mathcal{H}}_{1}(6)$. We now demonstrate that this class of systems support spatially compact energy-renormalized eigenstates of an arbitrary number $M$ of interacting particles. The intuitive idea is that as the interaction $\hat{\mathcal{H}}_{1}$ enforces particles to move in pairs between different unit cells, it prevents the motion of unpaired particles located in different unit cells. However, if, e.g., two particles sit in two distinct cells $n$ and $n+l$ with $1 \leqslant l \leqslant R$, then these particles interact, renormalizing the eigenenergy while remaining caged in their respective unit cells. Here $R$ is the interaction range of $\hat{\mathcal{H}}_{1}$ (6) defined as $R=\max _{n, m}|n-m|$ over all the pairs $n, m$ appearing in (6). A similar argument holds in higher dimensions.

More formally, we fix a set of distinct unit cells $n_{l}, l=$ $1, \ldots, M$ and consider a finite number $M$ of particles each located at a distinct unit cell $n_{l}$. This generates a $v^{M}$ subspace of the lattice's Hilbert space spanned by the basis vectors:

$$
\left|\psi_{\mathbf{a}}\right\rangle=\prod_{l=1}^{M} \hat{d}_{n_{l}, a_{l}}^{\dagger}|0\rangle,
$$

where $\mathbf{a}=\left(a_{1}, \ldots, a_{M}\right)$ is an $M$-dimensional integer vector specifying the position $a_{l}$ of the particle $l$ inside the unit cell $n_{l}$. Each of the above basis states is by construction an eigenstate of the operators $\hat{C}_{m}$ (7):

$$
\hat{C}_{m}\left|\psi_{\mathbf{a}}\right\rangle= \begin{cases}-\left|\psi_{\mathbf{a}}\right\rangle, & m=n_{l}, \\ \left|\psi_{\mathbf{a}}\right\rangle, & m \neq n_{l} .\end{cases}
$$

On the other hand, the subspace $X_{M}$ of the full Hilbert space spanned by all the states $\left|\psi_{\mathbf{a}}\right\rangle$ is an invariant subspace of the full Hamiltonian: by construction, any of $\left|\psi_{\mathbf{a}}\right\rangle$ is an eigenstate of $\hat{\mathcal{H}}_{\mathrm{fd}}$, the $\hat{\mathcal{H}}_{p}(6)$ vanishes on any of the $\left|\psi_{\mathbf{a}}\right\rangle$ due to single occupancy, while $\hat{\mathcal{H}}_{\mathrm{dd}}(6)$ can only couple different states 
$\left|\psi_{\mathbf{a}}\right\rangle$ to each other. There are two possibilities related to the distribution of the unit cells $n_{l}$ :

(i) $\left|n_{i}-n_{j}\right|>R$ for all $1 \leqslant i, j \leqslant M$, then the interaction $\hat{\mathcal{H}}_{1}$ vanishes for every basis state $\left|\psi_{\mathbf{a}}\right\rangle$. Therefore, they are all exact caged eigenstates of the full Hamiltonian $\hat{\mathcal{H}}$ with eigenenergy given by the sum of single-particle eigenenergies, e.g., FB energies, $E_{c}=\sum_{l} E_{a_{l}}$-hence $\left|\psi_{\mathbf{a}}\right\rangle$ is nonrenormalized.

(ii) At least one pair of particles is within the interaction range, e.g., the first two $1 \leqslant\left|n_{2}-n_{1}\right| \leqslant R$-then the interaction $\hat{\mathcal{H}}_{1}$, namely $\hat{\mathcal{H}}_{\text {dd }}$, couples those pairs, and in general $\left|\psi_{\mathbf{a}}\right\rangle$ is no longer an exact eigenstate of $\hat{\mathcal{H}}$.

In the latter case (ii), a number $\mathcal{P}(M)$ of configurations are possible where the interacting particles form $1 \leqslant s \leqslant S$ clusters with $\ell_{s}$ particles each-namely, $1 \leqslant\left|n_{i+1}-n_{i}\right| \leqslant R$ for $x_{s-1}+1 \leqslant i<x_{s}$; while $\left|n_{x_{s}+1}-n_{x_{s}}\right|>R$ for all $s$, with $x_{s}=\sum_{j \leqslant s} \ell_{j}$ and $x_{0}=1$-where $\mathcal{P}$ is the integer partition function [40]. The energy of these eigenstates is renormalized as $E(U)=\sum_{l} E_{a_{l}}+\sum_{s=1}^{S} \varphi_{s}(U)$ with functions $\varphi_{s}$ satisfying $\varphi_{s}(U \rightarrow 0)=0 . E(U)$ depends on the interaction $\hat{\mathcal{H}}_{1}$, the number of particles $M$, the number of disjoint clusters $S$, and how many particles $\ell_{s}$ reside within each cluster. We refer to such eigenstates as renormalized caged states.

We note that the eigenstates of the Hamiltonian $\hat{\mathcal{H}}$ within this invariant subspace are spatially compact as they are formed by $M$ unpaired particles locked in distinct unit cells by the conservation laws expressed by the operators $\hat{C}_{n}$. Therefore, due to the translation invariance of $\hat{\mathcal{H}}$, these families of compact eigenstates for any finite $M$ over an infinite chain $N \rightarrow \infty$ are macroscopically degenerate at each given $U$. Moreover, the above considerations apply to lattices $\hat{\mathcal{H}}$ of any spatial dimension, and energy-renormalized caged states are also expected for any $d \geqslant 2$. In that case, renormalized caged states of interacting particles can be arranged into diverse geometrical profiles, e.g., lines, loops, zigzags, patches, and strips, among others.

Below we illustrate the above results for the $d=1$ setting with a nearest-neighbor interaction $\hat{\mathcal{H}}_{1}$. Generalization to longer ranges or higher dimensions is straightforward.

\section{A. Two particles}

For $M=2$ particles, the invariant subspace $X_{2}$ is $v^{2}$ dimensional, $\mathbf{a}=(a, b)$, and the basis states are $\left|\psi_{a b}\right\rangle=$ $\hat{d}_{n, a}^{\dagger} \hat{d}_{m, b}^{\dagger}|0\rangle$ for any $1 \leqslant a, b \leqslant v$. There are three possibilities:

(i) $n=m$, the two particles pair up and can travel, giving rise to delocalized eigenstates of paired particles $[34,36]$.

(ii) $|n-m| \geqslant 2$, the two particles are caged and do not interact, corresponding to nonrenormalized caged eigenstates, e.g., CLS.

(iii) $|n-m|=1$, the two particles are caged and interact with each other, producing renormalizes caged states.

We focus on this last case: The Hamiltonian $\hat{\mathcal{H}}$ restricted over the subspace $X_{2}$ yields eigenstates of two caged interacting particles. They are also eigenstates of $\hat{C}_{n}$ (7), with $c_{n}=c_{n+1}=-1$ and $c_{m}=1$ for $m \neq n, n+1$. Among the resulting $v^{2}$ eigenenergies, $\mu_{2} \leqslant v^{2}$ are renormalized by $\hat{\mathcal{H}}_{1}$ and are continuous functions of $U$ (the number $\mu_{2}$ depends on the chosen $\left.\hat{\mathcal{H}}_{1}\right)$. In the noninteracting limit $U \rightarrow 0$, the eigenstates reduce to the basis vectors $\left|\psi_{a b}\right\rangle$ of $X_{2}$ and the renormalized energies become simply $E_{a}+E_{b}$.

These energy-renormalized two-particle caged statesdiscussed in Ref. [36] in the context of the ABF diamond chain with the Hubbard interaction-are macroscopically degenerate and their number scales as $N$ due to translation invariance.

\section{B. Three particles and beyond}

For $M=3$ the subspace $X_{3}$ is $v^{3}$-dimensional, $\mathbf{a}=$ $(a, b, c)$, and the basis states are given by $\left|\psi_{a b c}\right\rangle=$ $\hat{d}_{n, a}^{\dagger} \hat{d}_{n+1, b}^{\dagger} \hat{d}_{n+2, c}^{\dagger}|0\rangle$. The renormalized caged states emerge from two possible configurations:

(i) Two particles sitting in two adjacent cells, while the cell of the third particle is beyond the interaction range away from the interacting pair, e.g., $|\psi\rangle=\hat{d}_{n, a}^{\dagger} \hat{d}_{n+1, b}^{\dagger} \hat{d}_{m, c}^{\dagger}|0\rangle$ with $m \leqslant n-2$ or $m \geqslant n+3$.

(ii) Three particles in three adjacent cells, e.g., $|\psi\rangle=$ $\hat{d}_{n, a}^{\dagger} \hat{d}_{n+1, b}^{\dagger} \hat{d}_{n+2, c}^{\dagger}|0\rangle$.

Case (i) is a straightforward extension of the two-particle case presented in Sec. IV A: there are $\nu \mu_{2}$ renormalized caged states of this type, whose degeneracy scales as $N^{2}$. In case (ii) the eigenstates of the Hamiltonian $\hat{\mathcal{H}}$ restricted over the subspace $X_{3}$ are three-particles caged states of the system. They are also eigenstates of $\hat{C}_{n}$ with $c_{m}=-1$ for $n \leqslant m \leqslant n+2$ and $c_{m}=1$ otherwise. Among all the eigenenergies, $\mu_{3} \leqslant v^{3}$ are continuous functions of $U$ and their degeneracy scales as $N$. In the noninteracting limit $U=0$, these eigenenergies reduce to $E_{a}+E_{b}+E_{c}$ while the corresponding eigenstates become $\left|\psi_{a b c}\right\rangle$.

For larger $M$, the renormalized caged eigenstates emerge from $\mathcal{P}$ different configurations-a number growing as $\mathcal{P}(M) \sim e^{\sqrt{M}} / M$ for diverging $M$ [41]. Since the caged states have single unit-cell occupation, these states necessarily lose compactness and span over the entire infinite ABF network in the limit $M \rightarrow \infty$.

\section{An example}

We visualize the above results by explicitly computing the renormalized two-particle states for a sample one-dimensional $v=2 \mathrm{ABF}$ lattice given by the Hamiltonian $\hat{\mathcal{H}}=\hat{\mathcal{H}}_{0}+\hat{\mathcal{H}}_{1}$,

$$
\begin{gathered}
\hat{\mathcal{H}}_{0}=-\sum_{n \in \mathbb{Z}}\left[\frac{1}{2}\left(\hat{c}_{n}^{\dagger T} H_{0} \hat{c}_{n}\right)+\left(\hat{c}_{n}^{\dagger T} H_{1} \hat{c}_{n+1}\right)+\text { H.c. }\right], \\
\hat{\mathcal{H}}_{1}=\frac{U}{2} \sum_{n \in \mathbb{Z}}\left[\hat{a}_{n}^{\dagger} \hat{a}_{n}^{\dagger} \hat{a}_{n} \hat{a}_{n}+\hat{b}_{n}^{\dagger} \hat{b}_{n}^{\dagger} \hat{b}_{n} \hat{b}_{n}\right],
\end{gathered}
$$

showing that our results generalize those introduced in Ref. [36] in the context of the ABF diamond chain. The annihilation $\hat{c}_{n}=\left(\hat{a}_{n}, \hat{b}_{n}\right)$ and creation operators $\hat{c}_{n}^{\dagger}=\left(\hat{a}_{n}^{\dagger}, \hat{b}_{n}^{\dagger}\right)$ respect the commutation relations $\left[\hat{a}_{n}, \hat{a}_{k}^{\dagger}\right]=\delta_{n, k},\left[\hat{b}_{n}, \hat{b}_{k}^{\dagger}\right]=$ $\delta_{n, k}$, and $\left[\hat{a}_{n}, \hat{b}_{k}^{\dagger}\right]=0$ for any $n, k \in \mathbb{Z}$. The hopping matrices

$$
\begin{gathered}
H_{0}=\Gamma_{0}\left(\begin{array}{cc}
\left|z_{1}\right|^{2}-\left|w_{1}\right|^{2} & -2 z_{1} w_{1} \\
-2 z_{1}^{*} w_{1}^{*} & \left|w_{1}\right|^{2}-\left|z_{1}\right|^{2}
\end{array}\right), \\
H_{1}=\Gamma_{1}\left(\begin{array}{cc}
z_{1} w_{1}^{*} & z_{1}^{2} \\
-\left(w_{1}^{*}\right)^{2} & -z_{1} w_{1}^{*}
\end{array}\right)
\end{gathered}
$$



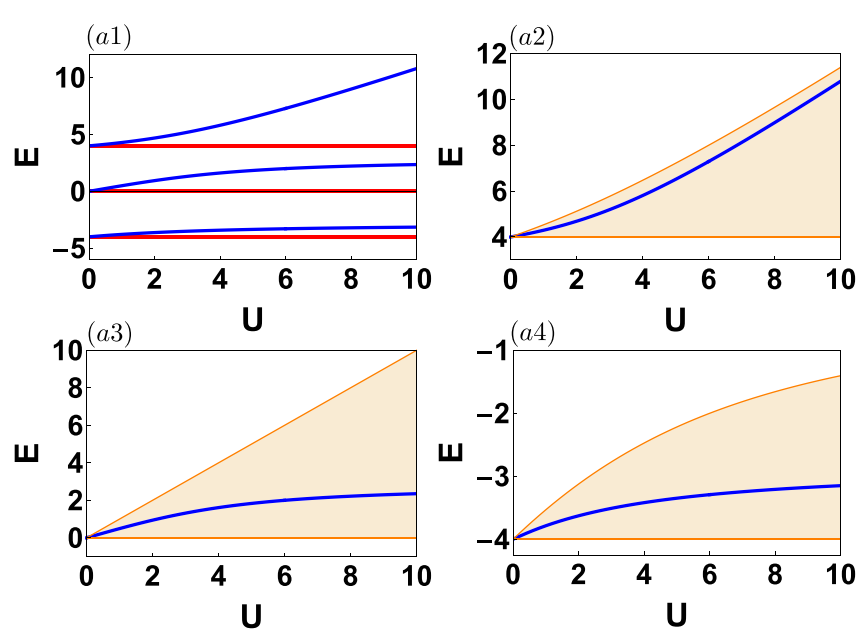

FIG. 1. (a1) Nonrenormalized degenerate energies (red lines) of two noninteracting CLS and renormalized energies (blue curves) of two compactly bound interacting particles versus $U$. (a2)-(a4) Renormalized energies (blue curve) within the dispersive bands (orange areas) of 2IP bound states.

describe hopping inside a unit cell and between the nearestneighbor unit cells, respectively; $\Gamma_{0}=\left|w_{2}\right|^{2}-\left|z_{2}\right|^{2}$ and $\Gamma_{1}=$ $2 z_{2} w_{2}$. Here $z_{i}, w_{i}$ are complex numbers such that $\left|w_{i}\right|^{2}+$ $\left|z_{i}\right|^{2}=1$ for $i=1,2$, and the Hamiltonian $\hat{\mathcal{H}}_{0}$ for a single particle $M=1$ has two flatbands at $E_{1,2}= \pm 1$ [28]. We show in Appendix B that for $\left|z_{1}\right|^{2}=\left|w_{1}\right|^{2}$ the Hamiltonian $\hat{\mathcal{H}}$ in Eqs. (8) and (9) can be recast as $\hat{\mathcal{H}}=\hat{\mathcal{H}}_{\mathrm{fd}}+\hat{\mathcal{H}}_{1}$ with a fully detangled noninteracting part and interaction in Eq. (6).

For $M=2$ particles, the Hamiltonian $\hat{\mathcal{H}}_{0}$ possesses four flatbands at $E_{j}=0, \pm 2$, with the zero-energy band being doubly degenerate. In the fine-tuned case $\left|z_{1}\right|^{2}=\left|w_{1}\right|^{2}$, two particles placed in two adjacent unit cells $n, n+1$ define a four-dimensional invariant vector subspace $X_{2}$ of the full Hilbert space spanned by the vectors

$$
\begin{array}{ll}
|1\rangle=\hat{\alpha}_{n}^{\dagger} \hat{\alpha}_{n+1}^{\dagger}|0\rangle, & |2\rangle=\hat{\alpha}_{n}^{\dagger} \hat{\beta}_{n+1}^{\dagger}|0\rangle, \\
|3\rangle=\hat{\alpha}_{n+1}^{\dagger} \hat{\beta}_{n}^{\dagger}|0\rangle, & |4\rangle=\hat{\beta}_{n}^{\dagger} \hat{\beta}_{n+1}^{\dagger}|0\rangle,
\end{array}
$$

where the creation and annihilation operators $\hat{\alpha}_{n}^{\dagger}, \hat{\beta}_{n}^{\dagger}, \hat{\alpha}_{n}, \hat{\beta}_{n}$ are expressed in the fully detangled representation $\hat{\mathcal{H}}_{\text {fd }}$ of $\hat{\mathcal{H}}_{0}$. As was discussed in Sec. IV A, the eigenstates of the Hamiltonian $\hat{\mathcal{H}}$ applied to the vector subspace $X_{2}$ correspond to renormalized caged states. For, e.g., the Creutz lattice $[29,34]$ - the case obtained for $z_{i}=\cos \pi / 4, w_{i}=\sin \pi / 4$ for $i=1,2$ in Eqs. (10) and (11), which we rescale by a factor of 2 turning the flatbands for $M=2$ particles at $E_{j}=0, \pm 4-$ the energies of these states are encoded in the characteristic polynomial (see Appendix C)

$$
\begin{aligned}
p_{F}(E ; U) & =E^{3}\left(E^{2}-16\right)\left(E^{3}-U E^{2}-16 E+8 U\right) \\
& \equiv E^{3}\left(E^{2}-16\right) g_{F}(E ; U) .
\end{aligned}
$$

This polynomial yields one nonrenormalized energy $E=0$ and three renormalized ones given by the zeros of $g_{F}(E ; U)$ energies shown in Fig. 1(a1) as a function of the interaction strength $U$ with red horizontal lines (nonrenormalized) and blue curves (renormalized). This polynomial is analogous
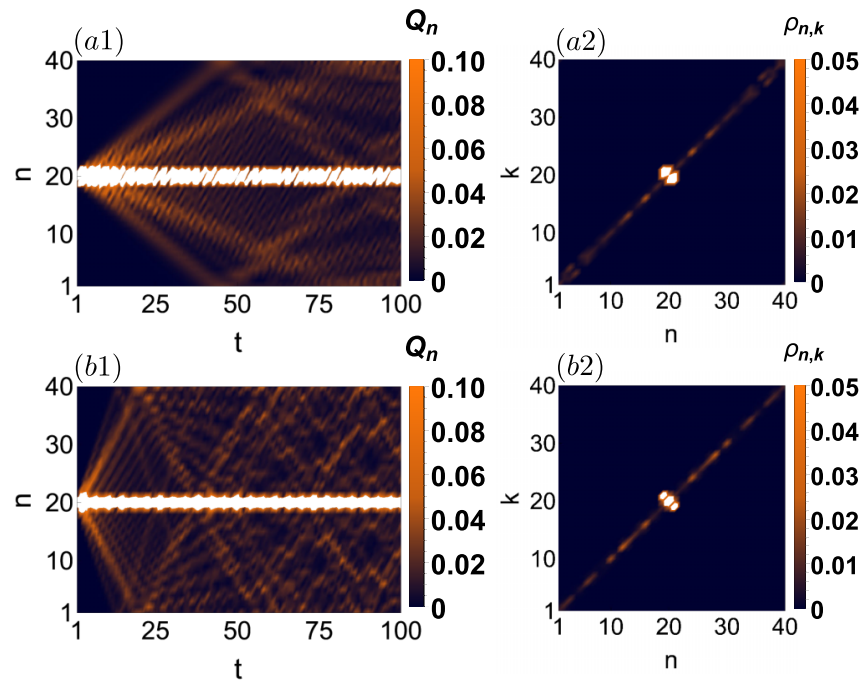

FIG. 2. Model A combined with the Hubbard interactionnetwork possessing the conserved quantities (7). (a1) Time evolution of $Q_{n}$ and (b1) local density $\rho_{n, k}$ at time $t=100$, both for $U=1$. (a2), (b2) Same as (a1), (b1) for $U=5$.

to that obtained for two interacting spinful fermions in the $\mathrm{AB}$ diamond chain [36], testifying that our results generalize the previous observations. In Figs. 1(a2)-1(a4) we plot each renormalized degeneracy shown in panel (a1) and one of the three dispersive bands (orange shaded areas) corresponding to extended states of paired particles [corresponding to case (i) in Sec. IV A] obtained as zeros of $g_{D}(E, k ; U)$ in

$$
\begin{aligned}
p_{D}(E, k ; U) & =E\left[E^{3}-U E^{2}-16 E+8 U(1-\cos k)\right] \\
& \equiv E g_{D}(E, k ; U) .
\end{aligned}
$$

In this case, we observe that all the renormalized energies (blue curves) lie within one of the dispersive bands-as for any $U$ it holds that $g_{F}(E, U)=g_{D}(E, 0 ; U)$ —characterizing these renormalized compact states as quantum two-particle bound states in the continuum (BIC) [42].

These renormalized caged states can have a nontrivial impact on the dynamics of $M$ interacting particles. Indeed, this already emerges in the two-particle case. Without loss of generality, we consider distinguishable bosons and we compute the time evolution of their wave function $|\Psi\rangle$ by evolving the associated two-dimensional Schrödinger lattice whose coordinates $(n, k)$ represent the spatial position of each boson (see Appendix D). We consider a network for $N=40$ unit cells and compute the local density $\rho_{n, k}$ of the two particles and the corresponding one-dimensional probability distribution function (PDF) of the particle density defined as $Q_{n}=\sum_{k=1}^{N} \rho_{n, k}$.

As the single-particle Hamiltonian $\hat{\mathcal{H}}_{0}$ testbed respecting the fine-tuning condition $\left|z_{1}\right|^{2}=\left|w_{1}\right|^{2}$, which ensures the existence of the conserved quantities (7), we consider $z_{1}=$ $\cos \pi / 4, w_{1}=\sin \pi / 4$ and $z_{2}=\cos \pi / 6, w_{2}=\sin \pi / 6$ in Eqs. (10) and (11), which is the quantum version of model $A$ studied in Ref. [28]. In Figs. 2(a1)-2(b1) we plot the time evolution of $Q_{n}$ for model $A$ for two interaction strengths $U=1$ (top) and $U=5$ (bottom), respectively. Both cases show that a part of the PDF is propagating ballisticallyindicating the spreading of the particle pair along the network, 

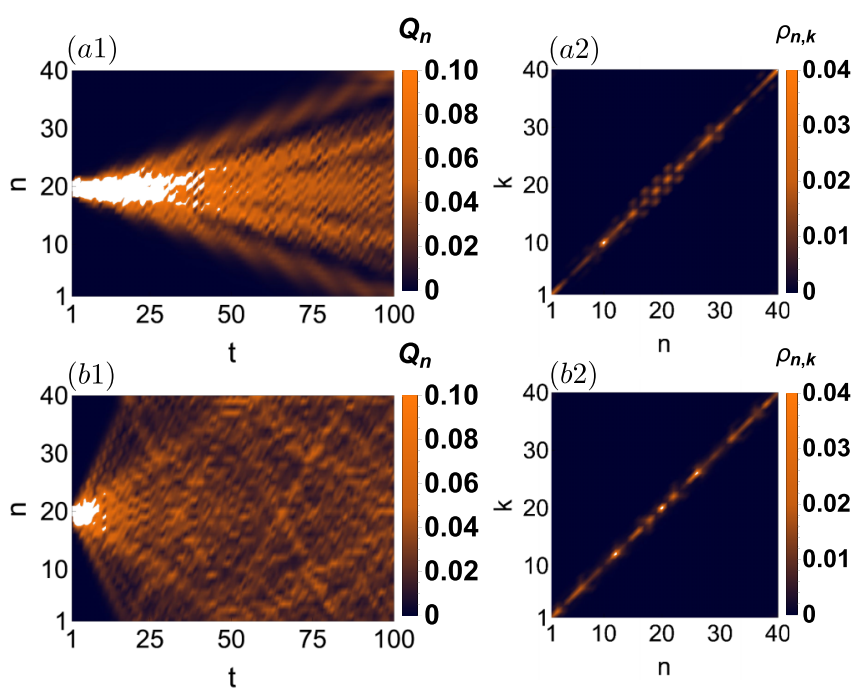

FIG. 3. Model B combined with the Hubbard interactionnetwork not possessing the conserved quantities (7). (a1) Time evolution of $Q_{n}$ and (b1) local density $\rho_{n, k}$ at time $t=100$, both for $U=1$. (a2), (b2) Same as (a1), (b1) for $U=5$.

breaking single-particle caging. Simultaneously, we observe that a substantial portion of $Q_{n}$ remains localized at unit cell $n=\frac{N}{2}$ (the initial location of both particles) due to overlap between the initial condition and the renormalized caged states. This is further detailed in Figs. 2(a2)-2(b2), where we plot the local density $\rho_{n, k}$ at time $t=100$ for $U=1$ and 5, respectively. Indeed, these panels show a large-amplitude density peak at the original launching cell, while the delocalized fraction of $\rho_{n, k}$ spread along the diagonal $k=n$ indicating that the particles have to stay bound in order to delocalize.

On the other hand, these effects vanish if the finetuning condition $\left|z_{1}\right|^{2}=\left|w_{1}\right|^{2}$ is violated and consequently both the renormalized caged states and conserved quantities $\hat{C}_{n}$ are absent. Let us consider, e.g., $z_{1}=\cos \pi / 6, w_{1}=$ $\sin \pi / 6$ and $z_{2}=\cos \pi / 4, w_{2}=\sin \pi / 4$ in Eqs. (10) and (11), the quantum version of model $B$ studied in Ref. [28]. In Figs. 3(a1)-3(b1) we show the time evolution of $Q_{n}$ for $U=1$ (top) and $U=5$ (bottom), respectively. These plots again show ballistic spreading. However, no considerable localized fraction of the PDF $Q_{n}$ is observed. This is also confirmed in Figs. 3(a2)-3(b2), where the local density $\rho_{n, k}$ at time $t=100$ is shown for $U=1$ and 5 , respectively.

\section{CONCLUSIONS AND PERSPECTIVES}

In this work, we showed that the quantum versions of classical nonlinear models exhibiting caging discussed in Ref. [28] are the only systems to feature an extended set of conserved quantities - number parity operators, first introduced in Ref. [34] for specific ABF geometries. Transport in these systems is realized only through moving pairs of interacting particles, while single unpaired particles remain caged. Next we demonstrated that the picture is more subtle: we explicitly showed the existence of macroscopically degenerate interaction-renormalized compact states for any finite number $M$ of particles on an infinite ABF lattice $N \rightarrow \infty$ generalizing their first observation for two spinful fermions in the $A B$ diamond chain [36]. These caged many-body states generalize the extensively studied and experimentally observed single-particle compact localized states [7-9], and hint toward quantum caging phenomena in interacting systems; see, e.g., Ref. [43] for the AB diamond chain. Notably, as these renormalized caged states coexist in the spectrum with extended eigenstates of paired particles (characterized by volume-law entanglement), they are examples of quantum many-body scars-states introduced in Refs. [44-48] and also discussed in the context of flatband networks in Refs. [49-51] - leading to weak ergodicity breaking phenomena $[47,52]$. Moreover, it appears that $M=2$-particle caged states are bound states in the continuum [42] —as their renormalized energies reside within dispersive bands of delocalized states of pairs of particles. Conjecturing this for any finite number of particles $M$ yields that these systems constitute a potential platform for the experimental realization of BICs in quantum systems [42].

The quantum caging of interacting particles highlights the all-bands-flat networks as a promising platform for novel phenomena in quantum many-body physics. An important front is the fate of these states at the finite-density limit and their impact on the many-body dynamics. While the interaction in general allows paired particles to freely move along the system [34] forbidding MBL_an obstacle that can be overcome by fine-tuning the interaction [37-39] - these states might contribute to anomalous thermalization phenomena. Thus the quest to search for anomalous many-body dynamics can not only be extended to interacting ABF lattices, including disorder, dissipation and external fields, but also to a lattice Hamiltonian supporting both flat and dispersive bands [53].

\section{ACKNOWLEDGMENTS}

The authors thank Ihor Vakulchyk, Ajith Ramachandran, Arindam Mallick, and Tilen Čadez for helpful discussions. This work was supported by the Institute for Basic Science, Korea (IBS-R024-D1).

\section{APPENDIX A: PROOF OF THE COMMUTATION OF THE PARITY OPERATORS WITH THE HAMILTONIAN}

We provide in this Appendix the formal proof that the parity operators (7) commute with the Hamiltonians (1) and (6). We assume that we work in the detangled basis of the single-particle Hamiltonian, e.g., the basis in which the singleparticle Hamiltonian is diagonal in the Fock space. Since the parity operators (7) are defined in that basis, and are diagonal in it, they commute with the single-particle Hamiltonian (1).

Let us consider a Fock state $\left|\ldots ; N_{n}, a_{n} ; \ldots\right\rangle$-with $N_{n}$ particles in unit cell $n$. The index $a_{n}$ enumerates all the possible partitionings of $N_{n}$ particles among the $v$ sites of the unit cell $n$. Such states form a complete basis of the Fock space. Next we write the interaction (6) as

$$
\begin{aligned}
\hat{\mathcal{H}}_{1}= & \frac{U}{2} \sum_{n a, b ; m c, d} V_{n a, n b ; m c, m d} \hat{d}_{n, a}^{\dagger} \hat{d}_{n, b}^{\dagger} \hat{d}_{m, c} \hat{c}_{m, d} \\
& +\frac{U}{2} \sum_{n a, b ; m c, d} V_{n a, m b ; n c, m d} \hat{d}_{n, a}^{\dagger} \hat{d}_{m, b}^{\dagger} \hat{d}_{n, c} \hat{d}_{m, d} .
\end{aligned}
$$


We define

$$
\begin{aligned}
& \hat{h}_{n a b ; m c d}=V_{n a, n b ; m c, m d} \hat{d}_{n, a}^{\dagger} \hat{d}_{n, b}^{\dagger} \hat{d}_{m, c} \hat{d}_{m, d}, \\
& \hat{g}_{n m ; a b c d}=V_{n a, m b ; n c, m d} \hat{d}_{n, a}^{\dagger} \hat{d}_{m, b}^{\dagger} \hat{d}_{n, c} \hat{d}_{m, d} .
\end{aligned}
$$

We prove that $\left[\hat{C}_{k}, \hat{h}_{n a, n b ; m c, m d}\right]\left|\ldots ; N_{m}, a_{m} ; \ldots\right\rangle=0$ and $\left[\hat{C}_{k}, \hat{g}_{n a, n b ; m c, m d}\right]\left|\ldots ; N_{m}, a_{m} ; \ldots\right\rangle=0$ for any $k$ and all the states $a_{m}$, and therefore also the commutator $\left[\hat{C}, \hat{\mathcal{H}}_{1}\right]=0$. In general,

$$
\hat{h}_{n a b ; m c d}\left|\ldots ; N_{m}, a_{m} ; \ldots\right\rangle=\sum_{b_{m}} x_{b_{m}}\left|\ldots ; N_{m}, b_{m} ; \ldots\right\rangle+\sum_{c_{m}, d_{n}} y_{c_{m}, d_{n}}\left|\ldots ; N_{m}-2, c_{m} ; \ldots ; N_{n}+2, d_{n} ; \ldots\right\rangle
$$

since $\hat{h}$ can only move pairs of particles between unit cells. Then for $k \neq n, m$ we have (for brevity, we omit the ... in the ket-states)

$$
\begin{aligned}
& \hat{h}_{n a b ; m c d} \hat{C}_{k}\left|N_{m}, a_{m}\right\rangle=(-1)^{N_{k}}\left[\sum_{b_{m}} x_{b_{m}}\left|N_{m}, b_{m}\right\rangle+\sum_{c_{m}, d_{n}} y_{c_{m}, d_{n}}\left|N_{m}-2, c_{m} ; N_{n}+2, d_{n}\right\rangle\right], \\
& \hat{C}_{k} \hat{h}_{n a b ; m c d}\left|N_{m}, a_{m}\right\rangle=(-1)^{N_{k}}\left[\sum_{b_{m}} x_{b_{m}}\left|N_{m}, b_{m}\right\rangle+\sum_{c_{m}, d_{n}} y_{c_{m}, d_{n}}\left|N_{m}-2, c_{m} ; N_{n}+2, d_{n}\right\rangle\right] .
\end{aligned}
$$

For $k=m$ we find

$$
\begin{aligned}
& \hat{h}_{\text {nab;mcd }} \hat{C}_{m}\left|N_{m}, a_{m}\right\rangle=(-1)^{N_{m}}\left[\sum_{b_{m}} x_{b_{m}}\left|N_{m}, b_{m}\right\rangle+\sum_{c_{m}, d_{n}} y_{c_{m}, d_{n}}\left|N_{m}-2, c_{m} ; N_{n}+2, d_{n}\right\rangle\right], \\
& \hat{C}_{m} \hat{h}_{n a b ; m c d}\left|N_{m}, a_{m}\right\rangle=(-1)^{N_{m}} \sum_{b_{m}} x_{b_{m}}\left|N_{m}, b_{m}\right\rangle+(-1)^{N_{m}-2} \sum_{c_{m}, d_{n}} y_{c_{m}, d_{n}}\left|N_{m}-2, c_{m} ; N_{n}+2, d_{n}\right\rangle,
\end{aligned}
$$

and for $k=n$,

$$
\begin{aligned}
& \hat{h}_{n a b ; m c d} \hat{C}_{n}\left|N_{m}, a_{m}\right\rangle=(-1)^{N_{n}}\left[\sum_{b_{m}} x_{b_{m}}\left|N_{m}, b_{m}\right\rangle+\sum_{c_{m}, d_{n}} y_{c_{m}, d_{n}}\left|N_{m}-2, c_{m} ; N_{n}+2, d_{n}\right\rangle\right], \\
& \hat{C}_{n} \hat{h}_{n a b ; m c d}\left|N_{m}, a_{m}\right\rangle=(-1)^{N_{n}} \sum_{b_{m}} x_{b_{m}}\left|N_{m}, b_{m}\right\rangle+(-1)^{N_{n}+2} \sum_{c_{m}, d_{n}} y_{c_{m}, d_{n}}\left|N_{m}-2, c_{m} ; N_{n}+2, d_{n}\right\rangle .
\end{aligned}
$$

In all three cases, the two expressions coincide. The case of $\hat{g}$ is treated identically to the above.

\section{APPENDIX B: ROTATING THE QUANTUM INTERACTION HAMILTONIAN $\hat{\mathcal{H}}_{1}$}

The Hubbard interaction Hamiltonian $\hat{\mathcal{H}}_{1}$ in Eq. (9) for $v=2$ reads

$$
\hat{\mathcal{H}}_{1}=\frac{U}{2} \sum_{n \in \mathbb{Z}}\left[\hat{a}_{n}^{\dagger} \hat{a}_{n}^{\dagger} \hat{a}_{n} \hat{a}_{n}+\hat{b}_{n}^{\dagger} \hat{b}_{n}^{\dagger} \hat{b}_{n} \hat{b}_{n}\right]
$$

The local unitary transformation that transforms the two-band ladder Eqs. (10) and (11) into decoupled dimers written for the operators $\hat{a}_{n}, \hat{b}_{n}$ and $\hat{p}_{n}, \hat{f}_{n}$ reads

$$
\begin{aligned}
& \hat{a}_{n}=e^{i \theta_{1}}\left(z_{1} \hat{p}_{n}+w_{1} \hat{f}_{n}\right), \\
& \hat{b}_{n}=e^{i \theta_{1}}\left(-w_{1}^{*} \hat{p}_{n}+z_{1}^{*} \hat{f}_{n}\right) .
\end{aligned}
$$

Then, $\hat{\mathcal{H}}_{1}$ in Eq. (B1) becomes

$$
\begin{aligned}
\hat{\mathcal{H}}_{1}= & \frac{U}{2} \sum_{n}\left\{\left(\left|z_{1}\right|^{4}+\left|w_{1}\right|^{4}\right) \hat{p}_{n}^{\dagger} \hat{p}_{n}^{\dagger} \hat{p}_{n} \hat{p}_{n}\right. \\
& +2 z_{1}^{*} w_{1}\left(\left|z_{1}\right|^{2}-\left|w_{1}\right|^{2}\right) \hat{p}_{n}^{\dagger} \hat{p}_{n}^{\dagger} \hat{p}_{n} \hat{f}_{n} \\
& +2 z_{1}^{* 2} w_{1}^{2} \hat{p}_{n}^{\dagger} \hat{p}_{n}^{\dagger} \hat{f}_{n} \hat{f}_{n}
\end{aligned}
$$

$$
\begin{aligned}
& +2 z_{1} w_{1}^{*}\left(\left|z_{1}\right|^{2}-\left|w_{1}\right|^{2}\right) \hat{p}_{n}^{\dagger} \hat{f}_{n}^{\dagger} \hat{p}_{n} \hat{p}_{n} \\
& +8\left|z_{1}\right|^{2}\left|w_{1}\right|^{2} \hat{p}_{n}^{\dagger} \hat{f}_{n}^{\dagger} \hat{p}_{n} \hat{f}_{n} \\
& +2 z_{1}^{*} w_{1}\left(\left|w_{1}\right|^{2}-\left|z_{1}\right|^{2}\right) \hat{p}_{n}^{\dagger} \hat{f}_{n}^{\dagger} \hat{f}_{n} \hat{f}_{n} \\
& +2 z_{1}^{2} w_{1}^{* 2} \hat{f}_{n}^{\dagger} \hat{f}_{n}^{\dagger} \hat{p}_{n} \hat{p}_{n} \\
& +2 z_{1} w_{1}^{*}\left(\left|w_{1}\right|^{2}-\left|z_{1}\right|^{2}\right) \hat{f}_{n}^{\dagger} \hat{f}_{n}^{\dagger} \hat{p}_{n} \hat{f}_{n} \\
& \left.+\left(\left|z_{1}\right|^{4}+\left|w_{1}\right|^{4}\right) \hat{f}_{n}^{\dagger} \hat{f}_{n}^{\dagger} \hat{f}_{n} \hat{f}_{n}\right\} .
\end{aligned}
$$

The fine-tuning $\left|w_{1}\right|^{2}=\left|z_{1}\right|^{2}$ and the unit-cell redefinition $\hat{p}_{n} \rightarrow \hat{p}_{n} ; \hat{f}_{n} \rightarrow \hat{f}_{n+1}$ simplifies Eq. (B3) to

$$
\begin{aligned}
\hat{\mathcal{H}}_{1}= & U \sum_{n}\left\{\left|z_{1}\right|^{4}\left[\hat{p}_{n}^{\dagger} \hat{p}_{n}^{\dagger} \hat{p}_{n} \hat{p}_{n}+\hat{f}_{n}^{\dagger} \hat{f}_{n}^{\dagger} \hat{f}_{n} \hat{f}_{n}+4 \hat{p}_{n}^{\dagger} \hat{f}_{n+1}^{\dagger} \hat{p}_{n} \hat{f}_{n+1}\right]\right. \\
& \left.+z_{1}^{* 2} w_{1}^{2} \hat{p}_{n}^{\dagger} \hat{p}_{n}^{\dagger} \hat{f}_{n+1} \hat{f}_{n+1}+z_{1}^{*} w_{1}^{* 2} \hat{p}_{n} \hat{p}_{n} \hat{f}_{n+1}^{\dagger} \hat{f}_{n+1}^{\dagger}\right\} . \quad \text { (B4) }
\end{aligned}
$$

Equation (B4) transformed via a second local unitary transformation [54] written for the operators $\hat{p}_{n}, \hat{f}_{n}$ and $\hat{\alpha}_{n}, \hat{\beta}_{n}$,

$$
\begin{aligned}
& \hat{f}_{n}=e^{i \theta_{2}}\left(z_{2} \hat{\alpha}_{n}+w_{2} \hat{\beta}_{n}\right), \\
& \hat{p}_{n}=e^{i \theta_{2}}\left(-w_{2}^{*} \hat{\alpha}_{n}+z_{2}^{*} \hat{\beta}_{n}\right),
\end{aligned}
$$


belongs to the class of Hamiltonian in Eq. (6)—recalled as follows:

$$
\begin{aligned}
\hat{\mathcal{H}}_{1}= & \frac{U}{2} \sum_{n a, b ; m c, d} V_{n a, n b ; m c, m d} \hat{d}_{n, a}^{\dagger} \hat{d}_{n, b}^{\dagger} \hat{d}_{m, c} \hat{d}_{m, d} \\
& +\frac{U}{2} \sum_{n a, b ; m c, d} V_{n a, m b ; n c, m d} \hat{d}_{n, a}^{\dagger} \hat{d}_{m, b}^{\dagger} \hat{d}_{n, c} \hat{d}_{m, d} .
\end{aligned}
$$

Indeed, without reporting the cumbersome set of terms, let us recap:

(a) The first two terms $\hat{p}_{n}^{\dagger} \hat{p}_{n}^{\dagger} \hat{p}_{n} \hat{p}_{n}+\hat{f}_{n}^{\dagger} \hat{f}_{n}^{\dagger} \hat{f}_{n} \hat{f}_{n}$ in Eq. (B4) transformed via (B5) yield terms like those in the second line of Eq. (B6) for $m=n$.

(b) Likewise, the third term $\hat{p}_{n}^{\dagger} \hat{f}_{n+1}^{\dagger} \hat{p}_{n} \hat{f}_{n+1}$ in Eq. (B4) transformed via (B5) yields terms like those in the second line of Eq. (B6) for $m=n+1$.

(c) The fourth term $\hat{p}_{n}^{\dagger} \hat{p}_{n}^{\dagger} \hat{f}_{n+1} \hat{f}_{n+1}$ in Eq. (B4) and its Hermitian conjugate transformed via (B5) yield instead terms like those in the first line of Eq. (B6) for $m=n+1$.

\section{APPENDIX C: RENORMALIZED TWO-PARTICLE STATES}

Let us consider two unpaired particles caged in two neighboring unit cells-without loss of generality, $n=1,2$. In the fully detangled representation $\hat{\mathcal{H}}_{\mathrm{fd}}$ of the network Eqs. (8) and (9) in the operators $\hat{\alpha}_{n}^{\dagger}, \hat{\alpha}_{n}, \hat{\beta}_{n}^{\dagger}, \hat{\beta}_{n}$ [Eq. (B5)], this yields a four-dimensional vector space $X_{2}$ spanned by

$$
\begin{array}{ll}
|1\rangle=\hat{\alpha}_{1}^{\dagger} \hat{\alpha}_{2}^{\dagger}|0\rangle, & |2\rangle=\hat{\alpha}_{1}^{\dagger} \hat{\beta}_{2}^{\dagger}|0\rangle, \\
|3\rangle=\hat{\alpha}_{2}^{\dagger} \hat{\beta}_{1}^{\dagger}|0\rangle, \quad|4\rangle=\hat{\beta}_{1}^{\dagger} \hat{\beta}_{2}^{\dagger}|0\rangle .
\end{array}
$$

In the space in $\hat{\mathcal{H}}_{1}$ only those terms originating by the third component $\hat{p}_{n}^{\dagger} \hat{f}_{n+1}^{\dagger} \hat{p}_{n} \hat{f}_{n+1}$ in Eq. (B4) for $n=1$ apply, since those originating by the first two components as well as the last two in Eq. (B4) apply only if more than one particle sits within one unit cell.

In the sample case obtained for $z_{i}=\cos \pi / 4, w_{i}=\sin \pi / 4$ for $i=1,2$ in Eqs. (10) and (11), the transformation in Eq. (B5) yields an effective interaction $\hat{\mathcal{H}}_{1}^{e}$,

$$
\begin{aligned}
\hat{\mathcal{H}}_{1}^{e}= & U \hat{p}_{1}^{\dagger} \hat{f}_{2}^{\dagger} \hat{\beta}_{1} \hat{f}_{2} \\
= & \frac{U}{4}\left(\hat{\alpha}_{1}^{\dagger} \hat{\alpha}_{1} \hat{\alpha}_{2}^{\dagger} \hat{\alpha}_{2}-\hat{\alpha}_{1}^{\dagger} \hat{\alpha}_{1} \hat{\alpha}_{2}^{\dagger} \hat{\beta}_{2}-\hat{\alpha}_{1}^{\dagger} \hat{\alpha}_{1} \hat{\alpha}_{2} \hat{\beta}_{2}^{\dagger}+\hat{\alpha}_{1}^{\dagger} \hat{\alpha}_{1} \hat{\beta}_{2}^{\dagger} \hat{\beta}_{2}\right. \\
& +\hat{\alpha}_{1}^{\dagger} \hat{\beta}_{1} \hat{\alpha}_{2}^{\dagger} \hat{\alpha}_{2}-\hat{\alpha}_{1}^{\dagger} \hat{\beta}_{1} \hat{\alpha}_{2}^{\dagger} \hat{\beta}_{2}-\hat{\alpha}_{1}^{\dagger} \hat{\beta}_{1} \hat{\alpha}_{2} \hat{\beta}_{2}^{\dagger}+\hat{\alpha}_{1}^{\dagger} \hat{\beta}_{1} \hat{\beta}_{2}^{\dagger} \hat{\beta}_{2} \\
& +\hat{\alpha}_{1} \hat{\beta}_{1}^{\dagger} \hat{\alpha}_{2}^{\dagger} \hat{\alpha}_{2}-\hat{\alpha}_{1} \hat{\beta}_{1}^{\dagger} \hat{\alpha}_{2}^{\dagger} \hat{\beta}_{2}-\hat{\alpha}_{1} \hat{\beta}_{1}^{\dagger} \hat{\alpha}_{2} \hat{\beta}_{2}^{\dagger}+\hat{\alpha}_{1} \hat{\beta}_{1}^{\dagger} \hat{\beta}_{2}^{\dagger} \hat{\beta}_{2} \\
& \left.+\hat{\beta}_{1}^{\dagger} \hat{\beta}_{1} \hat{\alpha}_{2}^{\dagger} \hat{\alpha}_{2}-\hat{\beta}_{1}^{\dagger} \hat{\beta}_{1} \hat{\alpha}_{2}^{\dagger} \hat{\beta}_{2}-\hat{\beta}_{1}^{\dagger} \hat{\beta}_{1} \hat{\alpha}_{2} \hat{\beta}_{2}^{\dagger}+\hat{\beta}_{1}^{\dagger} \hat{\beta}_{1} \hat{\beta}_{2}^{\dagger} \hat{\beta}_{2}\right) .
\end{aligned}
$$

The $4 \times 4$ matrix $A_{1}=\left(a_{i, j}\right)$ with $a_{i, j}=\left\langle i\left|\hat{\mathcal{H}}_{1}^{e}\right| j\right\rangle$ for $1 \leqslant i$, $j \leqslant 4$ reads

$$
A_{1}=\frac{U}{4}\left(\begin{array}{rrrr}
1 & -1 & 1 & -1 \\
-1 & 1 & -1 & 1 \\
1 & -1 & 1 & -1 \\
-1 & 1 & -1 & 1
\end{array}\right)
$$

The single-particle Hamiltonian $\hat{\mathcal{H}}_{\mathrm{fd}}$ in the fully detangled representation

$$
\hat{\mathcal{H}}_{\mathrm{fd}}=2\left\{\hat{\alpha}_{1}^{\dagger} \hat{\alpha}_{1}+\hat{\alpha}_{2}^{\dagger} \hat{\alpha}_{2}-\hat{\alpha}_{1}^{\dagger} \hat{\alpha}_{1}-\hat{\alpha}_{2}^{\dagger} \hat{\alpha}_{2}\right\}
$$

whose corresponding matrix $A_{0}$ over the space $X_{2}$ reads

$$
A_{0}=\left(\begin{array}{llll}
4 & 0 & 0 & 0 \\
0 & 0 & 0 & 0 \\
0 & 0 & 0 & 0 \\
0 & 0 & 0 & 4
\end{array}\right)
$$

The resulting matrix

$$
A=A_{0}+A_{1}
$$

yields a characteristic polynomial

$$
\begin{aligned}
p_{F}(E ; U) & =E^{3}\left(E^{2}-16\right)\left(E^{3}-U E^{2}-16 E+8 U\right) \\
& \equiv E^{3}\left(E^{2}-16\right) g_{F}(E ; U)
\end{aligned}
$$

\section{APPENDIX D: TWO INTERACTING PARTICLES-ASSOCIATED SCHRÖDINGER LATTICE}

The wave function $|\psi\rangle$ of $M$ particles on the network described by $\hat{\mathcal{H}}$ in Eqs. (8) and (9) can be expanded as

$$
|\psi\rangle=\sum_{\mathbf{n} \in \mathbb{Z}^{M}} \varphi_{\mathbf{n}} \cdot\left|v_{\mathbf{n}}\right\rangle_{\mathbf{a}, \mathbf{b}}
$$

for $\varphi_{\mathbf{n}}$ a $2^{M}$-dimensional complex vector, and $\left|v_{\mathbf{n}}\right\rangle_{\mathbf{a}, \mathbf{b}}=$ $\left\{\left|v_{\mathbf{n}}^{s}\right\rangle_{\mathbf{a}, \mathbf{b}}\right\}_{s \leqslant 2^{M}}$ the Fock basis representing all possible particles configurations. Here $\mathbf{n}=\left(n_{1}, \ldots, n_{M}\right)$ is a multi-index, with each $n_{s}$ indicating the unit cell where the sth particle is located-either on the $a$ or the $b$ chain.

The wave function $|\psi\rangle$ is governed by an $M$-dimensional Schrödinger lattice obtained by unfolding the equation $i \partial_{t}|\psi\rangle=\hat{\mathcal{H}}|\psi\rangle$. For $M=2$ particles, this lattice reduces to a two-dimensional system,

$$
\begin{aligned}
i \dot{\varphi}_{n, k}= & {\left[A+U V_{2}\right] \varphi_{n, k}+T_{n} \varphi_{n+1, k}+T_{n}^{\dagger} \varphi_{n-1, k} } \\
& +T_{k} \varphi_{n, k+1}+T_{k}^{\dagger} \varphi_{n, k-1},
\end{aligned}
$$

with $\varphi_{n, k}=\left(X_{n, k}, Y_{n, k}, W_{n, k}, Z_{n, k}\right)^{T}$. The on-site matrix $A$ is

$A=2 \Gamma_{0}\left(\begin{array}{cccc}\left|z_{1}\right|^{2}-\left|w_{1}\right|^{2} & -z_{1} w_{1} & -z_{1} w_{1} & 0 \\ -z_{1}^{*} w_{1}^{*} & 0 & 0 & -z_{1} w_{1} \\ -z_{1}^{*} w_{1}^{*} & 0 & 0 & -z_{1} w_{1} \\ 0 & -z_{1}^{*} w_{1}^{*} & -z_{1}^{*} w_{1}^{*} & \left|w_{1}\right|^{2}-\left|z_{1}\right|^{2}\end{array}\right)$

while the hopping matrices $T_{n}, T_{k}$ are

$$
\begin{aligned}
T_{n} & =\Gamma_{1}\left(\begin{array}{cccc}
z_{1} w_{1}^{*} & 0 & z_{1}^{2} & 0 \\
0 & z_{1} w_{1}^{*} & 0 & z_{1}^{2} \\
-\left(w_{1}^{*}\right)^{2} & 0 & -z_{1} w_{1}^{*} & 0 \\
0 & -\left(w_{1}^{*}\right)^{2} & 0 & -z_{1} w_{1}^{*}
\end{array}\right), \\
T_{k} & =\Gamma_{1}\left(\begin{array}{cccc}
z_{1} w_{1}^{*} & z_{1}^{2} & 0 & 0 \\
-\left(w_{1}^{*}\right)^{2} & -z_{1} w_{1}^{*} & 0 & 0 \\
0 & 0 & z_{1} w_{1}^{*} & z_{1}^{2} \\
0 & 0 & -\left(w_{1}^{*}\right)^{2} & -z_{1} w_{1}^{*}
\end{array}\right)
\end{aligned}
$$

for $\Gamma_{0}=\left|w_{2}\right|^{2}-\left|z_{2}\right|^{2}$ and $\Gamma_{1}=2 z_{2} w_{2}$. The matrix $V_{2}$ encoding the interaction reads

$$
V_{2}=\left(\begin{array}{cccc}
\delta_{n, k} & 0 & 0 & 0 \\
0 & 0 & 0 & 0 \\
0 & 0 & 0 & 0 \\
0 & 0 & 0 & \delta_{n, k}
\end{array}\right) .
$$


[1] P. W. Anderson, Absence of diffusion in certain random lattices, Phys. Rev. 109, 1492 (1958).

[2] B. Kramer and A. MacKinnon, Localization: Theory and experiment, Rep. Prog. Phys. 56, 1469 (1993).

[3] D. M. Basko, I. L. Aleiner, and B. L. Altshuler, Metal-insulator transition in a weakly interacting many-electron system with localized single-particle states, Ann. Phys. 321, 1126 (2006).

[4] I. L. Aleiner, B. L. Altshuler, and G. V. Shlyapnikov, A finitetemperature phase transition for disordered weakly interacting bosons in one dimension, Nat. Phys. 6, 900 (2010).

[5] M. Pino, L. B. Ioffe, and B. L. Altshuler, Nonergodic metallic and insulating phases of Josephson junction chains, Proc. Natl. Acad. Sci. USA 113, 536 (2016).

[6] D. A. Abanin, E. Altman, I. Bloch, and M. Serbyn, Colloquium: Many-body localization, thermalization, and entanglement, Rev. Mod. Phys. 91, 021001 (2019).

[7] O. Derzhko, J. Richter, and M. Maksymenko, Strongly correlated flat-band systems: The route from Heisenberg spins to Hubbard electrons, Int. J. Mod. Phys. B 29, 1530007 (2015).

[8] D. Leykam, A. Andreanov, and S. Flach, Artificial flat band systems: From lattice models to experiments, Adv. Phys.: X 3, 1473052 (2018).

[9] D. Leykam and S. Flach, Perspective: Photonic flatbands, APL Photon. 3, 070901 (2018).

[10] A. Mielke, Ferromagnetic ground states for the Hubbard model on line graphs, J. Phys. A 24, L73 (1991).

[11] H. Tasaki, Ferromagnetism in the Hubbard Models with Degenerate Single-Electron Ground States, Phys. Rev. Lett. 69, 1608 (1992).

[12] R. G. Dias and J. D. Gouveia, Origami rules for the construction of localized eigenstates of the Hubbard model in decorated lattices, Sci. Rep. 5, 16852 (2015).

[13] L. Morales-Inostroza and R. A. Vicencio, Simple method to construct flat-band lattices, Phys. Rev. A 94, 043831 (2016).

[14] W. Maimaiti, A. Andreanov, H. C. Park, O. Gendelman, and S. Flach, Compact localized states and flat-band generators in one dimension, Phys. Rev. B 95, 115135 (2017).

[15] M. Röntgen, C. V. Morfonios, and P. Schmelcher, Compact localized states and flat bands from local symmetry partitioning, Phys. Rev. B 97, 035161 (2018).

[16] L. A. Toikka and A. Andreanov, Necessary and sufficient conditions for flat bands in $M$-dimensional $N$-band lattices with complex-valued nearest-neighbour hopping, J. Phys. A 52, 02LT04 (2018).

[17] W. Maimaiti, S. Flach, and A. Andreanov, Universal $d=1$ flat band generator from compact localized states, Phys. Rev. B 99, 125129 (2019).

[18] J. Vidal, R. Mosseri, and B. Douçot, Aharonov-Bohm Cages in Two-Dimensional Structures, Phys. Rev. Lett. 81, 5888 (1998).

[19] B. Douçot and J. Vidal, Pairing of Cooper Pairs in a Fully Frustrated Josephson-Junction Chain, Phys. Rev. Lett. 88, 227005 (2002).

[20] K. Fang, Z. Yu, and S. Fan, Photonic Aharonov-Bohm Effect Based on Dynamic Modulation, Phys. Rev. Lett. 108, 153901 (2012).

[21] S. Longhi, Aharonov-Bohm photonic cages in waveguide and coupled resonator lattices by synthetic magnetic fields, Opt. Lett. 39, 5892 (2014).
[22] O. V. Kibis, H. Sigurdsson, and I. A. Shelykh, Aharonov-Bohm effect for excitons in a semiconductor quantum ring dressed by circularly polarized light, Phys. Rev. B 91, 235308 (2015).

[23] M. Hasan, I. V. Iorsh, O. V. Kibis, and I. A. Shelykh, Optically controlled periodical chain of quantum rings, Phys. Rev. B 93, 125401 (2016).

[24] S. M. Zhang and L. Jin, Compact localized states and localization dynamics in the dice lattice, Phys. Rev. B 102, 054301 (2020).

[25] K. Fang, Z. Yu, and S. Fan, Realizing effective magnetic field for photons by controlling the phase of dynamic modulation, Nat. Photon. 6, 782 (2012).

[26] S. Mukherjee, M. Di Liberto, P. Öhberg, R. R. Thomson, and N. Goldman, Experimental Observation of Aharonov-Bohm Cages in Photonic Lattices, Phys. Rev. Lett. 121, 075502 (2018).

[27] S. Gladchenko, D. Olaya, E. Dupont-Ferrier, B. Douçot, L. B. Ioffe, and M. E. Gershenson, Superconducting nanocircuits for topologically protected qubits, Nat. Phys. 5, 48 (2009).

[28] C. Danieli, A. Andreanov, T. Mithun, and S. Flach, Nonlinear caging in all-bands-flat lattices, Phys. Rev. B 104, 085131 (2021).

[29] M. Creutz, End States, Ladder Compounds, and Domain-Wall Fermions, Phys. Rev. Lett. 83, 2636 (1999).

[30] S. Takayoshi, H. Katsura, N. Watanabe, and H. Aoki, Phase diagram and pair Tomonaga-Luttinger liquid in a Bose-Hubbard model with flat bands, Phys. Rev. A 88, 063613 (2013).

[31] M. Tovmasyan, E. P. L. van Nieuwenburg, and S. D. Huber, Geometry-induced pair condensation, Phys. Rev. B 88, 220510(R) (2013).

[32] M. Tovmasyan, S. Peotta, P. Törmä, and S. D. Huber, Effective theory and emergent SU(2) symmetry in the flat bands of attractive Hubbard models, Phys. Rev. B 94, 245149 (2016).

[33] J. Jünemann, A. Piga, S.-J. Ran, M. Lewenstein, M. Rizzi, and A. Bermudez, Exploring Interacting Topological Insulators with Ultracold Atoms: The Synthetic Creutz-Hubbard Model, Phys. Rev. X 7, 031057 (2017).

[34] M. Tovmasyan, S. Peotta, L. Liang, P. Törmä, and S. D. Huber, Preformed pairs in flat Bloch bands, Phys. Rev. B 98, 134513 (2018).

[35] J. Vidal, P. Butaud, B. Douçot, and R. Mosseri, Disorder and interactions in Aharonov-Bohm cages, Phys. Rev. B 64, 155306 (2001).

[36] J. Vidal, B. Douçot, R. Mosseri, and P. Butaud, Interaction Induced Delocalization for Two Particles in a Periodic Potential, Phys. Rev. Lett. 85, 3906 (2000).

[37] C. Danieli, A. Andreanov, and S. Flach, Many-body flatband localization, Phys. Rev. B 102, 041116(R) (2020).

[38] Y. Kuno, T. Orito, and I. Ichinose, Flat-band many-body localization and ergodicity breaking in the Creutz Ladder, New J. Phys. 22, 013032 (2020).

[39] T. Orito, Y. Kuno, and I. Ichinose, Exact projector Hamiltonian, local integrals of motion, and many-body localization with symmetry-protected topological order, Phys. Rev. B 101, 224308 (2020).

[40] The partition function $\mathcal{P}$ represents the number of possible ways of writing an integer $M$ as a sum of positive integers.

[41] G. H. Hardy and S. Ramanujan, Asymptotic formulae in combinatory analysis, Proc. London Math. Soc. s2-17, 75 (1918). 
[42] C. W. Hsu, B. Zhen, A. D. Stone, J. D. Joannopoulos, and M. Soljacic, Bound states in the continuum, Nat. Rev. Mater. 1, 16048 (2016).

[43] M. Di Liberto, S. Mukherjee, and N. Goldman, Nonlinear dynamics of Aharonov-Bohm cages, Phys. Rev. A 100, 043829 (2019).

[44] O. Vafek, N. Regnault, and B. A. Bernevig, Entanglement of exact excited eigenstates of the Hubbard model in arbitrary dimension, SciPost Phys. 3, 043 (2017).

[45] N. Shiraishi and T. Mori, Systematic Construction of Counterexamples to the Eigenstate Thermalization Hypothesis, Phys. Rev. Lett. 119, 030601 (2017).

[46] S. Moudgalya, S. Rachel, B. A. Bernevig, and N. Regnault, Exact excited states of nonintegrable models, Phys. Rev. B 98, 235155 (2018).

[47] C. J. Turner, A. A. Michailidis, D. A. Abanin, M. Serbyn, and Z. Papic, Weak ergodicity breaking from quantum many-body scars, Nat. Phys. 14, 745 (2018).
[48] A. Hudomal, I. Vasic, N. Regnault, M. Serbyn, and Z. Papic, Quantum scars of bosons with correlated hopping, Commun. Phys. 3, 99 (2020).

[49] O. Hart, G. De Tomasi, and C. Castelnovo, From compact localized states to many-body scars in the random quantum comb, Phys. Rev. Res. 2, 043267 (2020).

[50] P. A. McClarty, M. Haque, A. Sen, and J. Richter, Disorderfree localization and many-body quantum scars from magnetic frustration, Phys. Rev. B 102, 224303 (2020).

[51] Y. Kuno, T. Mizoguchi, and Y. Hatsugai, Flat band quantum scar, Phys. Rev. B 102, 241115(R) (2020).

[52] M. Serbyn, D. A. Abanin, and Z. Papić, Quantum many-body scars and weak breaking of ergodicity, Nat. Phys. 17, 675 (2021).

[53] S. Tilleke, M. Daumann, and T. Dahm, Nearest neighbour particle-particle interaction in fermionic quasi one-dimensional flat band lattices, Z. Naturforsch. A 75, 393 (2020).

[54] Steps that detangle each decoupled dimer of the rotated twoband ladder, Eqs. (10) and (11) via Eq. (B2). 